\title{
Steel slit shear walls with double- tapered links capable of condition assessment
}

\section{AUTHOR(S):}

Kurata, Masahiro; He, Liusheng; Nakashima, Masayoshi

\section{CITATION:}

Kurata, Masahiro ...[et al]. Steel slit shear walls with double-tapered links capable of condition assessment. Earthquake Engineering and Structural Dynamics 2015, 44(8): 1271-1287

\section{ISSUE DATE:}

2015-07

URL:

http://hdl.handle.net/2433/265275

\section{RIGHT:}

This is the peer reviewed version of the following article: [Steel slit shear walls with double-tapered links capable of condition assessment, Earthquake Engineering and Structural Dynamics, 44(8) 1271-1287], which has been published in final form at [https://doi.org/10.1002/eqe.2517]. This article may be used for non-commercial purposes in accordance with Wiley Terms and Conditions for Use of Self-Archived Versions. This article may not be enhanced, enriched or otherwise transformed into a derivative work, without express permission from Wiley or by statutory rights under applicable legislation. Copyright notices must not be removed, obscured or modified. The article must be linked to Wiley's version of record on Wiley Online Library and any embedding, framing or otherwise making available the article or pages thereof by third parties from platforms, services and websites other tha .. 


\title{
Steel slit shear walls with double-tapered links capable of condition assessment
}

\author{
Masahiro Kurata ${ }^{1}$, Liusheng $\mathrm{He}^{2}$, Masayoshi Nakashima ${ }^{1}$
}

${ }^{1}$ Disaster Prevention Research Institute, Kyoto Univ., Gokasho, Uji, Kyoto, Japan

${ }^{2}$ Dept. of Arch. and Arch. Sys., Grad. Sch. of Eng., Kyoto Univ., Kyodai-Katsura, Kyoto, Japan

\begin{abstract}
The concept of using a hysteretic damper as a condition assessment device that functions immediately after a damaging earthquake is realized by making use of the residual out-ofplane deformation of links that are arranged in slit shear walls. According to the proposed inspection procedure, the maximum drift ratio experienced by the slit wall is estimated based on the number of torsionally deformed links whose dimensions are determined so that the links would exhibit notable out-of-plane deformation at the target deformations. The adoption of a double-tapered shape for the links makes it possible to significantly increase the amount of their out-of-plane deformation. The relationship between the dimensions and the out-of-plane deformation of the links is established using numerical simulations. The effectiveness of the proposed condition assessment scenario is verified by using a series of cyclic-loading tests for individual links and groups of links. As a hysteretic damper, the strength and stiffness of the links predicted by design equations matched well with test results.
\end{abstract}

Keywords: Shear walls, steel plates, structural health monitoring, static test, finite element method

\section{INTRODUCTION}

In urban societies characterized by factors such as density, promptness and globalization, continuity of living and business activities is essential for society's survival. Many occurrences, both natural and social, are responsible for impeding this continuity. In many earthquake-prone countries such as Japan, damaging earthquakes are a major factor blocking normal living and business activities. Immediately after a large earthquake society and its inhabitants want to know the status of the environment and whether they can continue their daily activities or leave the space that they occupy. Structural health monitoring (SHM) and corresponding condition assessment is a promising technology to serve this purpose.

Numerous attempts have been made to develop this approach, and actual implementation has occurred in various places [e.g. 1-3]. However, seismic application of SHM to structures already built are still limited, with physical implementation only applied to the most critical structures such as long-span bridges and key industrial facilities. The reason for this is straightforward, that is, the cost of initial investment and the associated long-term 
maintenance is considered too great. However, without clear understanding of how well SHM works and how accurate it is, justification of cost is extremely difficult, and the lack of cost-benefit analysis is most likely one of the greatest barriers. Another is "unfamiliarity" with the devices germane to SHM, which together have resulted in a rather negative attitude among the practitioners engaged in the design and construction of structures. This attitude should not be overlooked when considering the adoption of SHM in the construction industry which is known to be conservative. This conservative approach also suggests a tactic for success in the use of SHM which is to use devices, elements, or components that have already been adopted in current construction techniques.

In seismic design, passive dampers have gained popularity in past decades and have been incorporated in many building constructions [e.g. 4-7]. Among various damper devices, hysteretic dampers that dissipate energy by the material yielding are most common, very likely because of a lesser cost burden and the familiarity developed with practical application examples. A notable feature of hysteretic dampers is that they are inserted carefully into the main frame (made of beams and columns) to activate energy dissipation earlier than the yielding of the frame. This means that deformation induced into the dampers is magnified relative to the floor drift.

The above background suggests the concept of the hysteretic damper functioning as an SHM sensor in addition to its own function of energy dissipation. If this is realized, the problem of cost and unfamiliarity will be greatly reduced, which in turn will lead to drastically increasing applications of SHM to standard, not just special, structures. Continuing with this idea, there is an interesting type of hysteretic damper named the slit shear wall. The concept of the slit shear wall is illustrated in Figure 1. It is made of a steel panel, has many diamond-shaped openings (manufactured using laser-cutting), and when the wall sustains in-plane shear deformation (named lateral drift), each segment, bounded by two adjacent openings, named a link, behaves as a flexural member at the point of inflection located mid-height. Each link yields and later involves out-of-plane buckling, and the summation of the energies dissipated in individual links equates to the energy dissipated

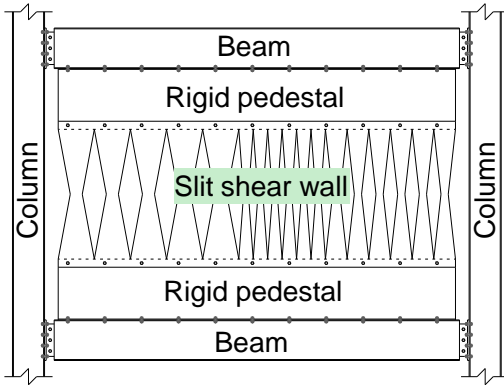

(a)

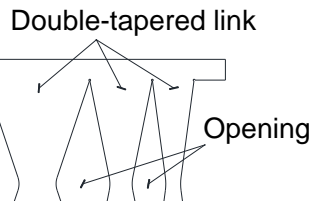

Link A B C

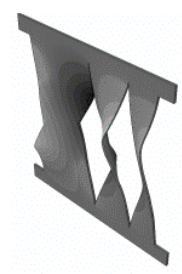

Isometric

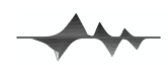

Top-down

(c)

Figure 1 Schematic diagram: (a) installation illustration; (b) a reference slit shear wall with double-tapered links; (c) deformation in simulation. 


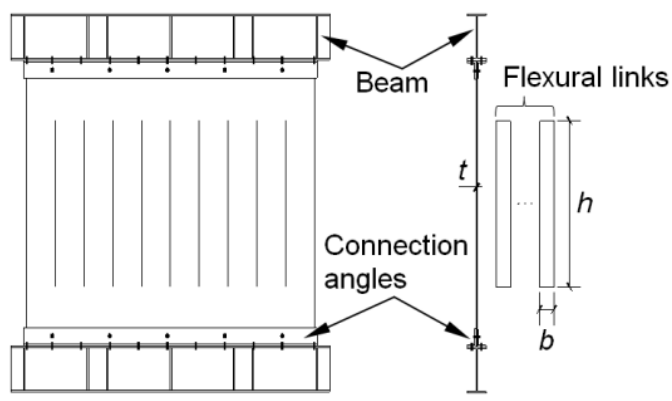

(a)

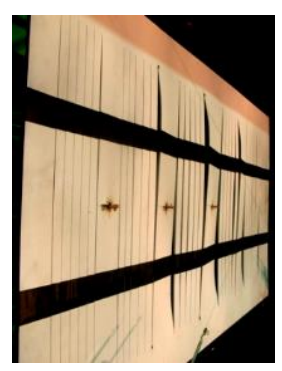

(b)

Figure 2 Slit shear wall with rectangular links: (a) assembly; (b) view from past test.

by the slit wall. The interesting features of the wall are that 1) the stiffness and strength of the wall can be adjusted flexibly by changing the slit arrangement, and 2) the wall dissipates energy with in-plane deformations of individual links, thereby not needing bracing by restrainers (often needed in steel plate shear walls).

The authors discovered a very interesting phenomenon associated with the deformation of individual links. As shown in Figure 1(c), each link exhibits torsional deformation after experiencing a certain level of inelastic cyclic lateral drift. Here torsional deformation is meant to be the out-of-plane rotation of the link caused by the buckling of the plate. Initiation of torsional deformation is controlled by the width of the link, i.e., earlier torsional deformation occurs in a wider link. The torsional deformation steadily increases for larger lateral drifts to a degree such that it is apparent to the eye. Enlightened by this interesting phenomenon, we came up with the following inspection procedure by which we can estimate the maximum lateral drift experienced by the slit shear wall.

Suppose the slit shear wall consists of three links of different widths (Figure 1(b)), with the widest link (Link A) exhibiting notable torsional deformation at a lateral drift ratio of $1 \%$ (the lateral drift divided by the link height), the second widest link (Link B) at a lateral drift ratio of $2 \%$, and the narrowest link (Link C) at a lateral drift ratio of $3 \%$. If no link shows notable torsional deformation, the maximum lateral drift ratio sustained by the wall is no greater than $1 \%$. If only Link A shows notable torsional deformation, the maximum lateral drift ratio is greater than $1 \%$ but smaller than $2 \%$. If Links A and B show notable torsional deformation, the maximum lateral drift ratio is between $2 \%$ and $3 \%$, and so forth.

This paper reports on 1) the design of links, named monitoring links, 2) numerical analysis to examine the relationship between the link's dimensions and its out-of-plane deformation, and corresponding torsional rotation, 3) a series of tests on individual links and groups of links, and 4) preliminary designs of slit walls that make it possible to estimate the maximum lateral drift by visual inspection.

\section{DESIGN OF MONITORING LINKS}

Shear wall systems using steel plates are very common in the field of earthquake 
engineering with their large stiffness, lightness, and ductility. Among the many types of steel shear walls, the special steel plate shear wall (SPSW) and slit shear wall are the most common in practice. The SPSW is widely accepted in North America and is included in design standards $[8,9]$. It resists shear deformation with tension field action after the onset of buckling and presents a slight pinching behavior in its hysteresis loop [e.g. 10-12]. Because a SPSW requires rigid plate boundaries, the four sides of the plate need to be fixed to stiff boundary members or an equivalent stiff boundary system [13]. Slit shear walls are fairly popular in Japan. As illustrated in Figure 2(a), each rectangular link behaves as a flexural member, and its yielding and hysteresis becomes a source of energy dissipation similar to conventional steel hysteresis dampers [e.g., 14]. Since Hitaka and Matsui introduced the design philosophy of slit shear walls, many studies, including practical applications to real buildings, have been reported [15-17]. In a slit shear wall, two major parameters control the energy dissipation behavior of each link, i.e., the width-thickness ratio (b/t in Figure 2(a), where $b$ is the link width and $t$ is the plate thickness) and the aspect ratio $(h / b$ in Figure 2(a), where $h$ is the height of the link). If the link is thin in $b / t$, local buckling occurs first and eventually out-of-plane deformations develop; if the link is long in $h / b$, yielding at the link ends occurs first, and the in-plane behavior dominates; but if the link is too long, the link becomes too flexible and its energy dissipation lessens significantly. According to previous studies [15-16] and actual implementation, feasible values for $b / t$ and $h / b$ are 8 to 24 and 3 to 10 , respectively, considering the balance between the dimensions and the strength desired in design. Note also that the slit walls adopted in real construction were 10 to $15 \mathrm{~mm}$ thick.

The authors previously attempted the development of monitoring links, whose details are given in [18-20]. In that development, slit shear walls shown in Figure 2(b), were designed and tested. A few links with different widths were arranged in the wall, and the wall and consequently the links, were loaded cyclically with increasing amplitude. The wider links buckled earlier, followed by the buckling of narrower links. In that study, the concept for monitoring using slit shear walls was found feasible, but the following two problems were identified. One was the degree of buckling, and the other was cracks initiated from the edge of the link. Although each segment exhibited buckling and corresponding out-of-plane deformation involving torsional deformation, the degree of the deformation was not necessarily significantly large, which had made it rather difficult to judge whether or not it sustained "notable" changes. As the link behaved as a flexural member, the maximum strain (and the corresponding plastic hinge) occurred at the end of the link, i.e., the slit edge. The large strain at the edge triggered cracking at that location, which in turn made the growth of torsional deformation unstable and inconsistent, and lessened the dissipation of energy.

Taking into account the outcomes of the past test, this study proposes a novel shape of monitoring links shown in Figure 1(b). The concept of a double-tapered steel component is not new [e.g., 21], and was used as a means of energy dissipation. Its effectiveness relative to its rectangular shape was achieved by controlling the locations of plastic hinges as a function of the rate of taper. However, the primary intention of the double-tapered shape 
proposed here is not the enhanced energy dissipation but the relocation of the plastic hinge away from the edge of the link, by which initiation and growth of the cracks will be avoided. Another, more important aspect of this shape is that the degree of torsional deformation can be significantly amplified relative to the conventional rectangular shape.

\section{DESIGN OF THE DOUBLE-TAPERED LINK}

\section{Configuration of the double-tapered link}

A schematic of a double-tapered link when subjected to an in-plane shear force $Q$ is illustrated in Figure 3(a), where $a, b, h$, and $t$ denote the link middle section width, end section width, height, and thickness. While the bending moment under in-plane shear becomes largest at the ends of the link, the link can start yielding at a location away from the ends as a result of its tapered shape.

The location of first yielding corresponds to the location of maximum bending stress. With the coordinate system shown in Figure 3(a), the bending stress along the link edge is calculated as:

$$
\sigma(x)=\frac{M(x) y}{I(x)}=\frac{3 M_{0}}{h t} \frac{1}{\left(\frac{b-a}{h} \sqrt{x}+\frac{a}{2 \sqrt{x}}\right)^{2}},
$$

where $M(x)$ is the bending moment at the cross-section whose ordinate is $x$, with $M_{0}$ the bending moment at the end section, and $I(x)$ the moment of inertia at the cross-section whose ordinate is $x$. The maximum bending stress is derived by solving the first order differential equation: $d \sigma / d x=0$, which leads to:

$$
\sigma_{\max }=\frac{3 M_{0}}{2 a t(b-a)}, \text { when } x=\frac{a h}{2(b-a)} .
$$

The location suitable for the maximum bending stress and corresponding yielding should be away from the end section. If it is too close to the mid-section, however, the strength and energy dissipation of the link becomes too small. As a compromise, one-quarter height from the mid-section was chosen as the location that sustains the maximum bending moment. Inserting $x=h / 4$ in Equation (2), $b / a=3$ is attained. This means that the link should be tapered so that the width of the mid-section is made $1 / 3$ the width at the end.

\section{Strength and stiffness of the double-tapered link}

Using the classical beam theory, the in-plane shear force that corresponds to the first yielding, named the yield strength $Q_{y}$, is:

$$
Q_{y}=\frac{8 a^{2} t}{3 h} \sigma_{y},
$$

where $\sigma_{y}$ is the yield stress.

Assuming that the plastic hinge is formed at the one-quarter height where the maximum 
172 strain occurs, the shear force that corresponds to the full plastic condition, named the plastic 173 strength $Q_{p}$, is:

$$
Q_{p}=1.5 Q_{y}=\frac{4 a^{2} t}{h} \sigma_{y} .
$$

Note, however, that the double-tapered link will sustain buckling, which in turn results in torsional deformation of the link. The strength of the link controlled by buckling will be explained later.

178 The elastic stiffness of a double-tapered link can be expressed as:

179

$$
K_{\text {link }}=\frac{1}{\int_{-h / 2}^{h / 2} \frac{M(x)^{2}}{E I(x)} d x+\int_{-h / 2}^{h / 2} \frac{Q^{2}}{G A(x)} d x}=\frac{1}{\frac{h^{3}}{12.7 E a^{3} t}+\frac{0.55 h}{G a t}},
$$

where $E$ is Young's modulus and $G$ is the shear modulus.

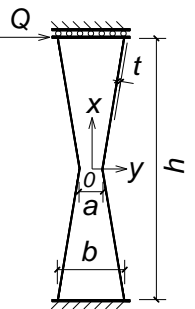

(a)

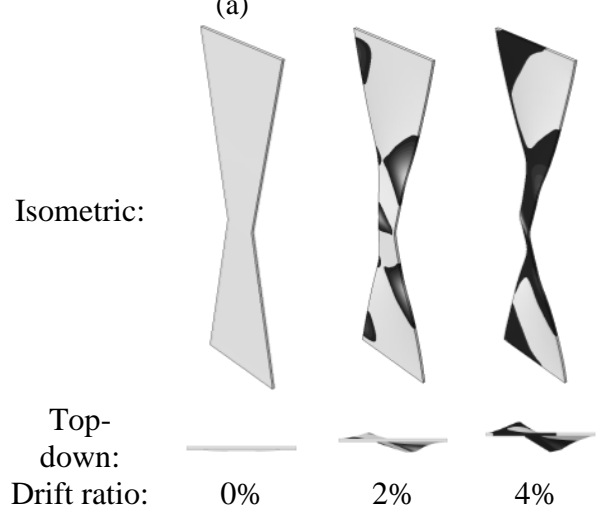

(c)

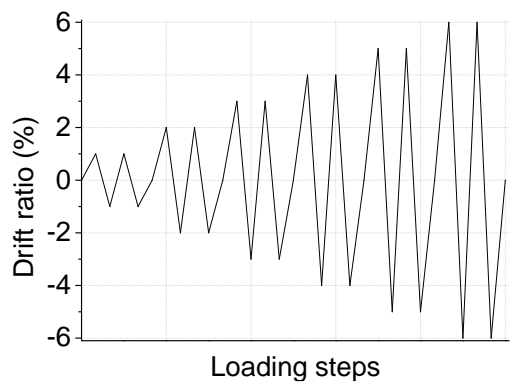

(b)

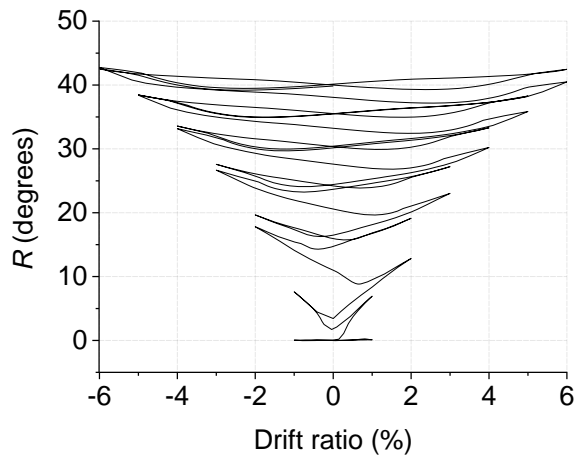

(d)

Figure 3 Double-tapered link: (a) schematic; (b) loading history; (c) progress of torsional deformation (dark color indicating yielding); (d) angle of rotation $(\lambda=22.2)$. 


\section{PRELIMINARY ANALYSIS}

Given the thickness of the link $t$ and the width ratio between the end and mid sections, $b / a=3$, the mid-section width $a$ and height $h$ formed the two major parameters that controlled the behavior of the double-tapered link. One parameter was the width-thickness ratio $\lambda$ and the other was the aspect ratio $\beta$. Because the cross-section at the one-quarter height would yield first and control the post-yielding behavior including buckling and the succeeding torsional deformation, the link width at the quarter height, $2 a$, instead of $a$, was taken as the reference width. Thus, the width-thickness ratio was defined as $\lambda=2 a / t$; and the aspect ratio as $\beta=h / 2 a$.

To understand the post-yielding behavior of the double-tapered link, preliminary analysis was conducted using the commercial finite element (FE) code, ABAQUS 6.10 [22]. In the FE model, a three-dimensional four-node shell element with reduced integration (S4R) was adopted to represent the link. The adopted mesh size allowed at least 10 seeds at the narrowest middle section. A displacement associated with the first mode was imposed on the FE model as the initial imperfection. In the direction normal to its plane, the maximum imperfection amplitude of the link was scaled to $1 / 500$ of the link height. Sensitivity analysis was conducted with respect to the mesh size and the amount of initial imperfection, and stable results were obtained with the values adopted. In the simulation, a yield stress of $374 \mathrm{MPa}$ and a strain hardening ratio of $0.5 \%$ were adopted for the material. Note that the yield stress was obtained from the coupon test conducted together with the cyclic loading tests whose details will be presented later.

Figure 3(b) shows the loading history used in the simulation. Displacement-controlled cyclic loading was applied to the top boundary of the link, while the bottom boundary was fixed. The amplitude of cyclic loading was increased incrementally from $1.0 \%$ to $6.0 \%$ with an increment of $1.0 \%$. Loading at the same drift ratios was repeated twice.

\section{Effect of width-thickness ratio}

In the thin plate theory, the width-thickness ratio controls local buckling. First, a parametric study on the width-thickness ratio was conducted to examine its effect on the behavior of the double-tapered link. In light of past research and practice, a $13.5 \mathrm{~mm}$ thick plate was considered, and three links with different widths at mid-section were chosen, i.e., $a=150,90$ and $60 \mathrm{~mm}$, which correspond to the width-thickness ratios of $\lambda=22.2,13.3$ and 8.9. For $\lambda=22.2$ as an example, Figure 3(c) shows the progress of out-of-plane deformation for incremental cyclic loading. The link buckled at a drift ratio of $1 \%$ from the yielded area at the quarter-height sections, torsional deformation of the link became notable at a drift ratio of $2 \%$, and the amount of torsional deformation increased during succeeding loading cycles. The middle part of the link bounded by one-quarter and three-quarter heights behaved nearly as a rigid body. The angle of rotation $(R)$ of the mid-section was used to quantify the amount of torsional deformation. Figure 3(d) shows the growth of the angle of rotation with respect to drift ratios. The rotation initiated at the drift ratio of $1 \%$ and increased consistently as loading progressed. In one loading cycle, the amplitude of torsional deformation was largest at the maximum drift ratio and decreased as the link was unloaded 
to zero displacement. This was natural because of the elastic component present at the maximum drift ratio. The ratio of reduction of torsional deformation caused by unloading was large right after the onset of buckling but became smaller with the increase in amplitude. This observation is important because the condition assessment of the wall will be performed after a major earthquake, in which residual inter-story drifts are commonly not so significant.

Figure 4(a) shows the growth of torsional deformation with respect to the drift ratio. The dotted lines with marks correspond to the angle measured after two cycles are completed at each amplitude, with the residual drift ratio of zero. It clearly shows that the initiation and growth of torsional deformation differed with $\lambda$. The values adopted here, i.e., $\lambda=22.2$, 13.3 and 8.9, were chosen to exhibit torsional deformation at the drift ratios of $1 \%, 2 \%$ and $4 \%$, respectively. The lower and upper solid lines correspond to the rotation angle at the residual drift ratio of $20 \%$ of the maximum drift ratio before and after the completion of two-cycle loading. For each width-thickness ratio, the differences among the three lines are very small, indicating that some residual deformation would change the torsional deformation very little.

\section{Effect of the aspect ratio}

Suppose the width and thickness of the link are specified, and the height of the link is the remaining parameter. If the link is too short, shear deformation (instead of flexural deformation) and yielding govern the behavior, which is not a preferred mode in the slit shear wall. To prevent early shear yielding at the narrowest mid-section, the lower bound of $\beta$ is taken as 4 in this study.

Conversely, if the link is too long, lateral torsional buckling is the likely failure mode [23]. It is impractical to derive a closed-form equation for the exact elastic buckling load of a double-tapered link because of the tapered shape. Bradford and Cuk [24] proposed an empirical equation for a web-tapped I-shaped cantilever subjected to a tip load, which is

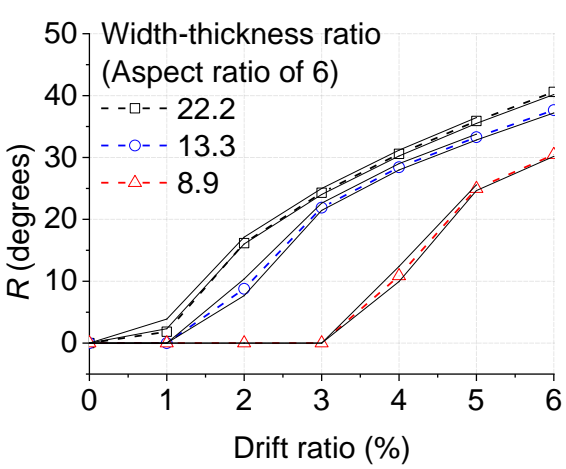

(a)

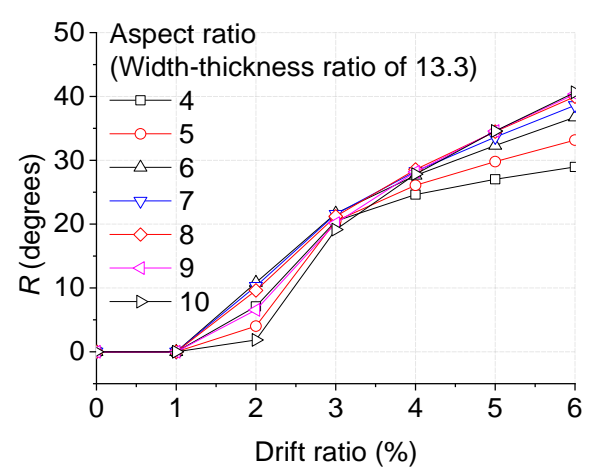

(b)

Figure 4 Angle of rotation $(R)$ versus drift ratio relationship for links: (a) effect of widththickness ratio; (b) effect of aspect ratio. 
equivalent to half of a double-tapered link. They estimated the critical load as:

$$
Q_{l t}=\frac{\eta \sqrt{\left(E I_{y} G J\right)_{0}}}{l^{2}},
$$

where $\mid$ is half length of the link, $\eta$ is the coefficient against the beam torsion parameter $k=\frac{\pi}{l} \sqrt{\left(\frac{E I_{w}}{G J}\right)_{0}}$, with $E I_{w}$ as the warping rigidity and $G J$ as the torsional stiffness, and the subscript 0 indicates the rigidity at the largest section.

An associated empirical equation for $\eta$ is as:

$$
\eta=3.24 k+3.94
$$

To study the effect of the aspect ratio, double-tapered links with a mid-section width of 90 $\mathrm{mm}$ and thickness of $13.5 \mathrm{~mm}$, were considered as the prototype links. For $\beta=10$ as an example, the elastic lateral torsional buckling load $Q_{l t}$ was $80.8 \mathrm{kN}$, while the load corresponding to the yield strength $Q_{y}$ was $60.6 \mathrm{kN}$. This means that the elastic lateral torsional buckling was not a dominant mode in promoting out-of-plane deformation. Following the equation, an aspect ratio $\beta$ of 12 was needed for the lateral torsional mode at the load of $Q_{y}$, which was greater than the aspect ratios, of between 3 and 10, commonly used for slit shear walls.

For the prototype double-tapered links, Figure 4(b) shows the increase in torsional deformation for various aspect ratios ranging from 4 to 10. For larger aspect ratios, the growth of rotation was somewhat slower, but overall the difference was considered minor compared with the difference observed for different width-thickness ratios.

\section{PREPARATION OF THE TEST}

\section{Test specimens}

The prototype slit shear wall was assumed to be arranged as illustrated in Figure 1, in which half of the story height was filled with a rigid element, and the drift ratio of the slit shear wall was then doubled. Assuming each story was $3 \mathrm{~m}$ high, the height of the slit shear wall was $1.5 \mathrm{~m}$. Considering the end zones needed for connections, the link height was taken to be $900 \mathrm{~mm}$, with a thickness of $13.5 \mathrm{~mm}$. In the test, scaled slit shear walls were tested for various link dimensions.

Ten specimen pairs were prepared with the major test variables adopted as: (1) the widththickness ratio; (2) the aspect ratio; and (3) the shape of the link. As discussed earlier, the width-thickness ratio was believed to be the primary factor that would control the out-ofplane deformation, and therefore three ratios were adopted, namely, $\lambda=23,14$ and 9 (the values slightly different with those used in simulation since the actual thickness of the tested plate was $4.3 \mathrm{~mm}$ instead of the nominal thickness of $4.5 \mathrm{~mm}$ ), which were targeted to exhibit notable torsional deformation at drift ratios of $1.5 \%$ (Level 1), 2.5\% (Level 2) and $3.5 \%$ (Level 3), respectively. Note that those width-thickness ratios were determined 
from the FE analysis introduced in the previous section.

Table 1 shows a list of the specimen pairs 1 to 10 . Pairs 1 to 4 were for Level 1, Pairs 5 to 8 were for Level 2, Pair 9 was for Level 3, and Pair 10 was for the combination of links for Level 1 to 3. Pairs 4 and 8 had rectangular links, with $b, \lambda$ and $\beta$ in Table 1 as the width, $\lambda=h / b$ and $\beta=b / t$, respectively. The number of links in each specimen was denoted as $n$. The word "Pair" was used for the specimen designation because two identical or nearly identical specimens were tested for each loading. For Level 1 and 2, links of two thicknesses, $2.2 \mathrm{~mm}$ and $4.3 \mathrm{~mm}$, were tested to demonstrate that the behavior would be similar for the same width-thickness ratio, and two heights, $300 \mathrm{~mm}$ and $200 \mathrm{~mm}$, were adopted to demonstrate that the aspect ratio was not a controlling factor. Pair 3 with an aspect ratio of 3 outside the recommendation, - of at least 4, and chesen to avoid shear yielding at the mid-section, was also included to verify the recommendation. To demonstrate effectiveness, i.e., more enhanced torsional deformation, achieved by the double-tapered links, conventional rectangular links were also tested. Finally, one more specimen was added, in which the three links assigned for Levels 1, 2 and 3 were installed in one slit shear wall. This last specimen was tested to verify the procedure to visually estimate the maximum drift ratio experienced by the wall.

Figure 5 shows the dimensions of the specimen. In Pairs 1, 2, 4 and 8, one specimen was made of a single plate in which three identical links were formed, while in the other specimen, three identical elements, each of which had just one link, were placed together as an assembly (Figure 5(a), (b), (d) and (h), respectively). This treatment was to observe whether the close boundaries between the adjacent links within a single plate would affect the torsional deformation behavior of individual links. The shaded region denotes the part contacted with two angles that served to fix the specimen to the loading setup (with a width of $60 \mathrm{~mm}$ ). The circles in the shaded region are the openings for bolting. The links were shaped by laser-cutting with a numerically controlled machine, which was found to be very useful and accurate. Mild steel SS400, a Japanese steel grade equivalent to A36, was used for the material of the tested double-tapered links. To determine the basic material

Table 1 Summary of the specimens

\begin{tabular}{|c|c|c|c|c|c|}
\hline Category & Pair & Link dimension $(\mathrm{mm})$ & $\lambda$ & $\beta$ & $c$ \\
\hline \multirow{4}{*}{ Level 1} & 1 & $a / t / h=25 / 2.2 / 300$ & 22.7 & 6 & 60 \\
\hline & 2 & $a / t / h=25 / 2.2 / 200$ & 22.7 & 4 & 110 \\
\hline & 3 & $a / t / h=50 / 4.3 / 300$ & 23.3 & 3 & 60 \\
\hline & 4 & $b / t / h=50 / 2.2 / 300$ & 22.7 & 6 & 60 \\
\hline \multirow{4}{*}{ Level 2} & 5 & $a / t / h=15 / 2.2 / 300$ & 13.6 & 10 & 60 \\
\hline & 6 & $a / t / h=15 / 2.2 / 200$ & 13.6 & 6.7 & 110 \\
\hline & 7 & $a / t / h=30 / 4.3 / 300$ & 14.0 & 5 & 60 \\
\hline & 8 & $b / t / h=30 / 2.2 / 300$ & 13.6 & 10 & 60 \\
\hline Level 3 & 9 & $a / t / h=20 / 4.3 / 300$ & 9.3 & 7.5 & 60 \\
\hline Combination & 10 & Combination of links & $\mathrm{s} 3,7 \mathrm{a}$ & & \\
\hline
\end{tabular}


311 properties, uniaxial tensile tests were performed, with an obtained average yield stress of $312374 \mathrm{MPa}$ and maximum stress of $440 \mathrm{MPa}$.

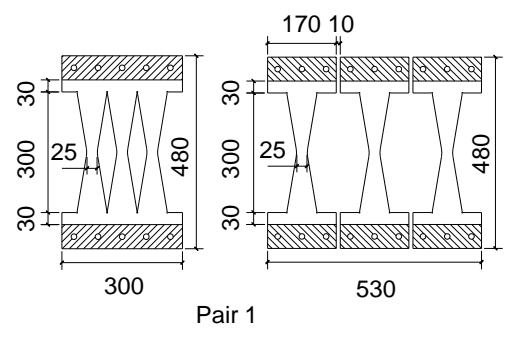

(a)

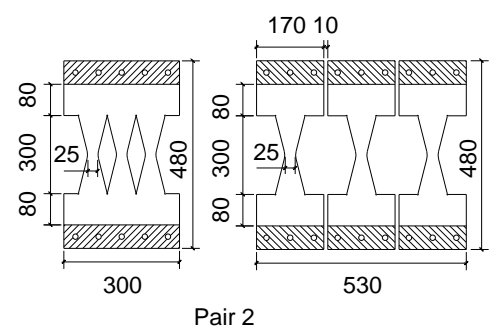

(b)

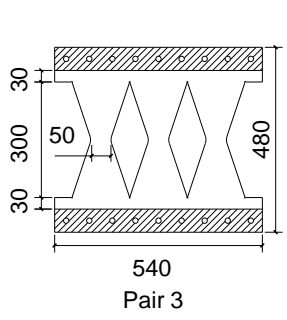

(c)

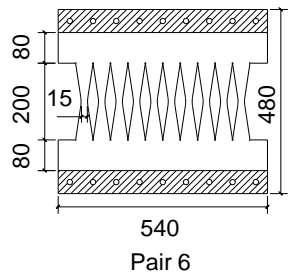

(f)

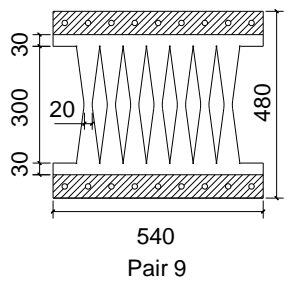

(i)

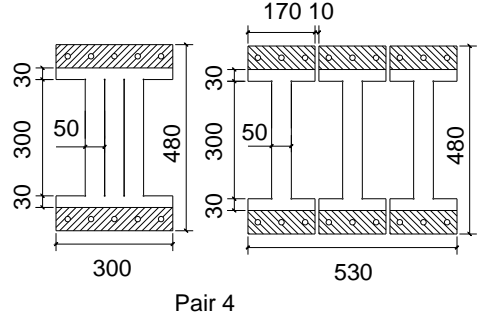

(d)

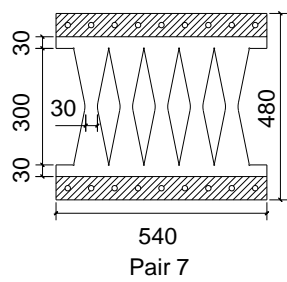

(g)

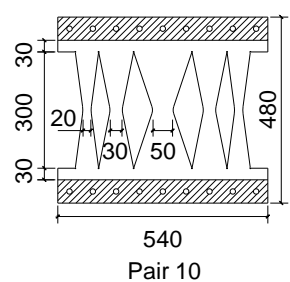

(j)

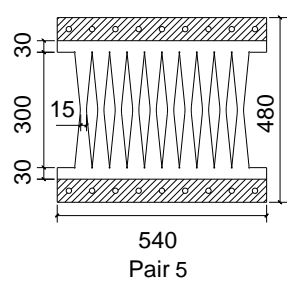

(e)

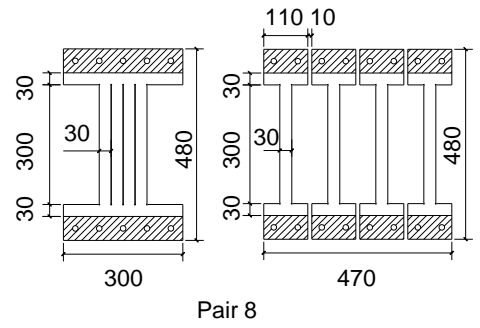

(h)

Figure 5 Details of specimens (unit: mm): (a) to (j) Pairs 1 to 10. 
In this study, notable torsional deformation was observed by visual inspection. Although this sounds rather subjective, notable torsional deformation was recognized very clearly once the out-of-plane displacement of the edges along the quarter-sections exceeded one thickness of the link as shown in Figure 6. This criterion of "off-one-thickness" corresponded to angles of rotation $(R)$ of $5^{\circ}, 8^{\circ}$ and $12^{\circ}$ for Level $1(\lambda=23)$, Level $2(\lambda$ $=14)$, and Level $3(\lambda=9)$, respectively.

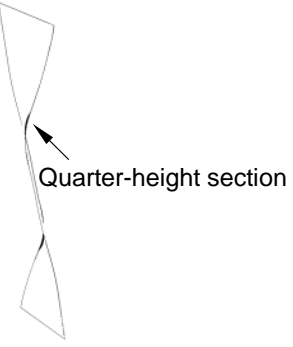

(a)

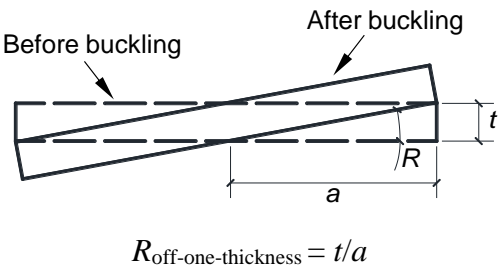

(b)

Figure 6 Definition of notable buckling definition: (a) torsional deformation after the onset of buckling; (b) "off-one-thickness" criterion and rotation angle calculation.

\section{Test setup and loading protocol}

The specimen was installed with a rotation of $90^{\circ}$ in a steel frame made of three wideflange columns $(\mathrm{H}-250 \times 250 \times 9 \times 14 \mathrm{~mm})$ as shown in Figure $7(\mathrm{a})$. The two exterior columns were securely posted on the base frame, while the middle column was attached to the vertical jack and moved vertically. To orient the middle column vertically, both ends of the middle column were clamped by restrainers and rollers. Two nearly identical specimens were installed as one pair, with one specimen installed on each side of the middle column to check the variability of the two seemingly identical specimens. The drift ratio, i.e., the

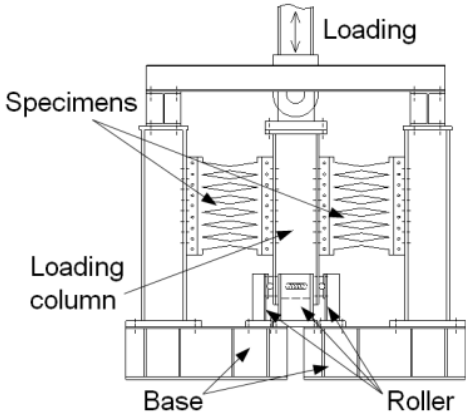

(a)

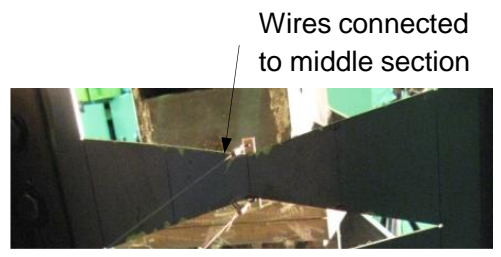

(b)

Figure 7 Test setup and measurement: (a) loading and specimen installation; (b) measurement of rotation angle. 
shear displacement, of the specimen, was controlled by the measurement of the vertical displacement of the middle column, and the shear force applied was measured by the load cell attached to the vertical jack. As the specimens on both sides were nearly identical, the shear force applied to each specimen was taken to be half the force detected by the load cell. Great care was taken to measure the torsional deformation of individual links, as shown in Figure 7(b). The torsional deformation was quantified as the rotation angle at the midsection. Two wires were connected to each link at the mid-section and perpendicular to the in-plane of the link, with the other two ends of wires connected to two displacement transducers.

The loading protocol adopted for preliminary FE analysis (Figure 3(b)) was slightly modified to increase the resolution of the amplitude corresponding to buckling. In addition to the basic drift ratios, i.e., $1 \%$ to $6 \%$ with increments of $1 \%$, a small increment of $0.5 \%$ was adopted near the expected drift ratio for buckling. For example, Level 1 (the target drift ratio of $1.5 \%$ ) specimens were subjected to cyclic loading with successive drift ratios of $0.5 \%, 1 \%, 1.5 \%, 2 \%, 3 \%, 4 \%, 5 \%$ and $6 \%$.

\section{TEST RESULTS}

\section{Yielding in a quarter-height section}

Yielding and local buckling at the quarter-height sections were the unique feature of the proposed double-tapered link. To carefully observe that behavior, several foil strain gauges that were to measure the elastic deformation were glued on the front face of the links at their quarter-height as shown in Figure 8(a) and (b). The gauges were glued in the longitudinal direction of the link and $2 \mathrm{~mm}$ inside its edge. Figure 8(b) shows the growth of the respective strains (the vertical axis) in relation to the cyclic drift ratio (the horizontal axis). The horizontal dashed lines indicate the strain corresponding to yielding. The plotted strain values were those corresponding to the first maximum displacement in the first cycle

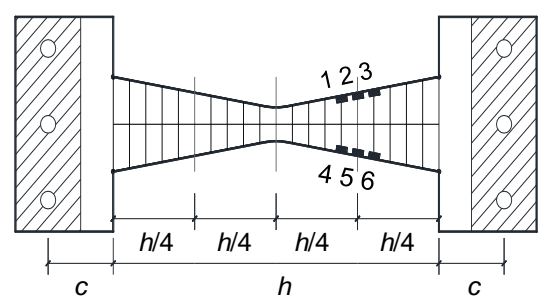

(a)

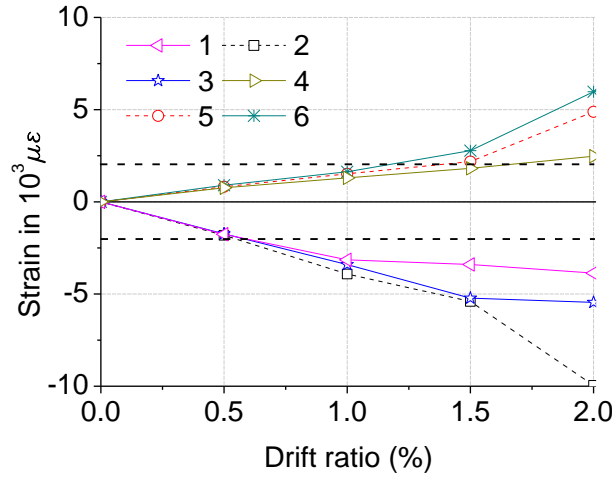

(b)

Figure 8 Strains at quarter-height sections: (a) location and numbering of strain gauges; (b) transition of strains. 
of respective cyclic amplitudes. Up to a drift ratio of $1.0 \%$, the strain values on the two sides were nearly the same but opposite in sign. This symmetrical pattern was violated for drift ratios above $1.5 \%$, when all strain gauges near the quarter-height section exhibited yielding, which eventually formed notable torsional deformation. This drift ratio of $1.5 \%$ corresponding to the initiation of notable titling will be discussed in the next section.

\section{Initiation and growth of torsional deformation}

Figure 9 shows the growth of torsional deformation (quantified by the angle of rotation $R$ ) with respect to the drift ratio. The vertical axis shows the angle of rotation observed at the completion (meaning zero drift ratio) of each amplitude with two cycles. Among Pairs 1 to 9, Pairs 3, 5, 6, 7 and 9 had identical specimens in each pair. In Pairs 1, 2, 4 and 8, the two specimens were slightly different, one as a single panel configured with links, while the other as an assembly of individual links. Comparison between the single panel specimen and individual link observed in Pairs 1, 2, 4 and 8, will be explained in the next section.

Figure 9(a) plots the four pairs in Level 1, in which all links have the same width-thickness ratio. Pairs 1 and 2 exhibited nearly the same behavior and reached a level of notable torsional deformation (according to the "off-one-thickness" criterion) at the drift ratio of $1.5 \%$ as expected prior to the test. The difference between Pair 1 and Pair 2 was the aspect ratio, $\beta$ (6 versus 4 ), and this test result verified the discussion that the aspect ratio would not be a controlling factor. Pair 3 had the same width-thickness ratio, but the torsional deformation was somewhat different.

This was attributed to the small aspect ratio adopted in the link, a value that was smaller than the recommended value of 4 . The resulting early shear yielding at the mid-section caused the middle part between the quarter-height sections to behave no longer as a rigid body and accordingly showed a different rotation. In Pair 4 with rectangular links, the initiation of torsional deformation was similar, but the growth of torsional deformation was significantly smaller than the corresponding double-tapered links. One of the motivations to adopt the double-tapered link was more significant torsional deformation, which was evidenced by the difference in the growth of torsional deformation between the $X$ shapeddouble-tapered and rectangular links.

Figure 9(b) plots the four pairs in Level 2, where the target drift ratio for notable buckling was $2.5 \%$. Pairs 5 and 6 with different aspect ratios reached the target angle of rotation at a drift ratio of $2.5 \%$, which means the torsional deformation was as expected. On the other hand, Pair 7 showed slightly earlier and greater torsional deformation than Pairs 5 and 6 . This was most likely caused by the difference in flexibility of the end zones; Pair 7 was made of a thicker plate than those for Pairs 5 and 6 . The influence of the end zone will be further discussed in the next section. Pair 8 was for rectangular links, and the torsional deformation progressed slower than the double-tapered links. This comparison again shows the advantage of the double-tapered link over the rectangular link in terms of visual inspection. Figure 9(c) plots the pair for Level 3, and, as expected, notable torsional deformation occurred at a drift ratio of $3.5 \%$. Thus, at all levels the test showed that the proposed double-tapered links exhibited notable torsional deformation at the target drift 
ratios.

\section{Difference in the angle of rotation of links within a specimen}

In Pairs $1,2_{-}$and 42 , one specimen was made of a single panel with three links configured, while the other specimen was an assembly of three individual links. Figure 9(d) shows the growth of the angle of rotation with respect to the drift ratio for Pair 1. The " 1 st" in Figure 10 -means the exterior link and " 2 nd" means the middle link. The results for Pairs 2 and 4 were similar. This closeness between the links in the panel and individual links, and between the exterior and middle links, indicated the independence of a link's torsional deformation even when neighbored by other links. It was also notable that there was little sensitivity to the growth of out-of-plane deformations once the shape was specified.

\section{Behavior of the panel for condition assessment}

Pair 10 was configured with a combination of three different double-tapered links, each representing Levels 1, 2 and 3. Link 1 in Figure 10(a) was the same as the links used for

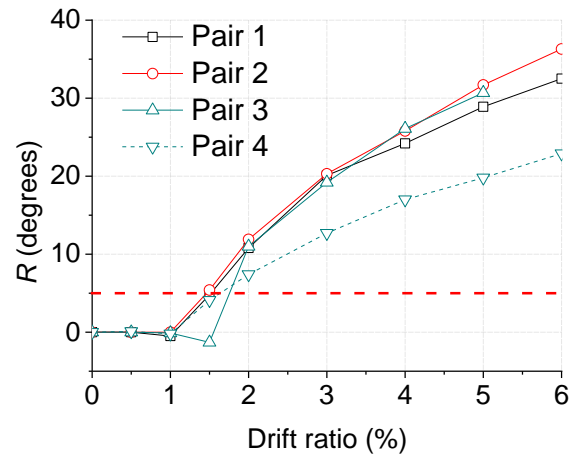

(a)

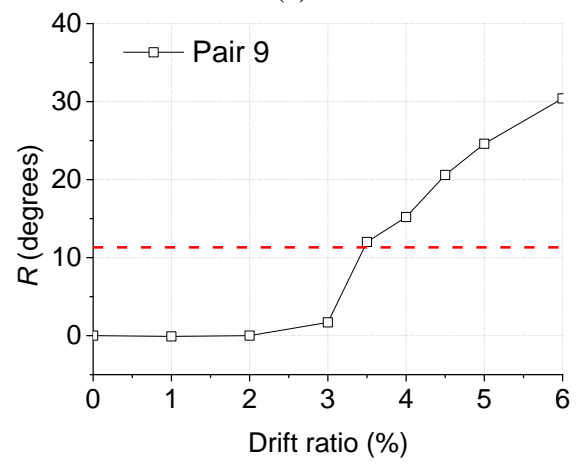

(c)

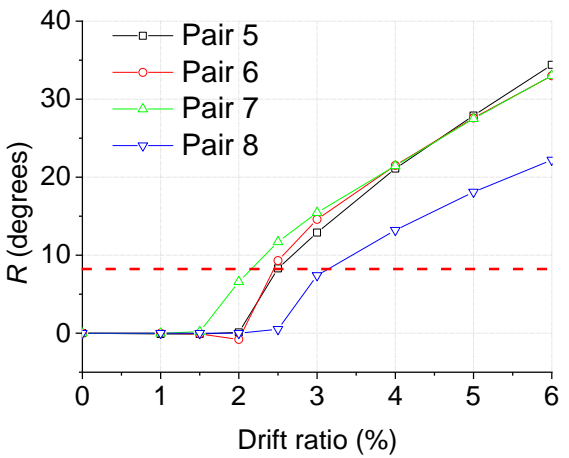

(b)

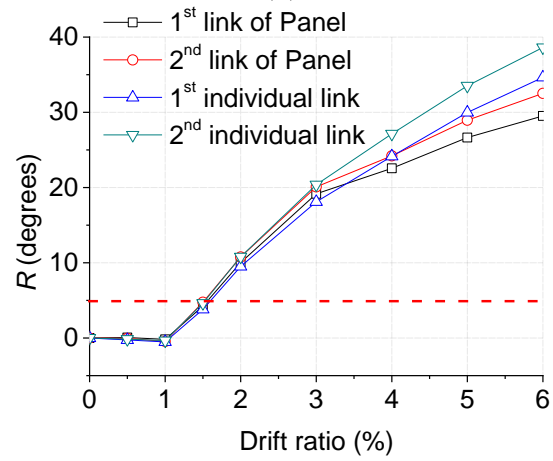

(d)

Figure 9 Angle of rotation $(R)$ versus drift relationship: (a) Level 1; (b) Level 2; (c) Level 3 ; (d) individual link versus panel for Pair 1. 


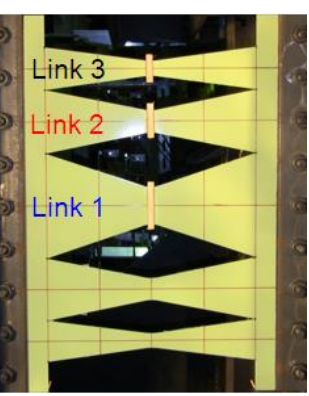

(a)

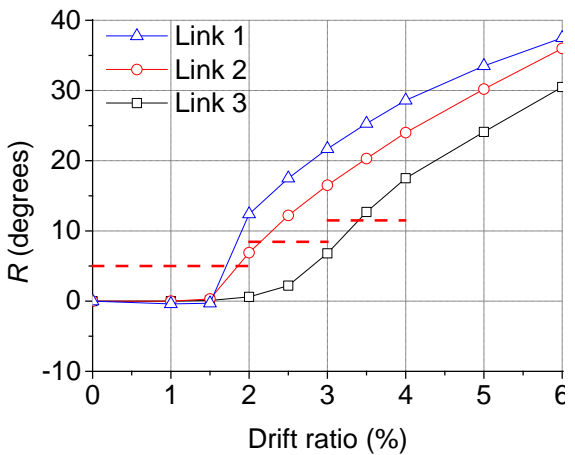

(b)

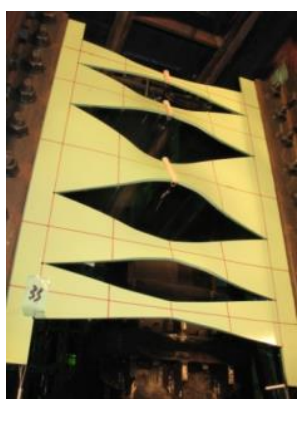

(c)

Figure 10 Behavior of Pair 10 with combination: (a) front view before loading; (b) angle of rotation $(R)$ versus drift ratio relationship; (c) upward view after 2 cycles of $3.5 \%$.

Pair 3 (Level 1), Link 2 was the same as the links used for Pair 7 (Level 2), and Link 3 was the same as the links used for Pair 9 (Level 3). The test results in Figure 10(b) clearly show that the actual behavior was nearly identical to that obtained in Pairs 3, 7 and 9 (Figure 9(a) to (c)), i.e., Links 1, 2 and 3 exhibited notable torsional deformation at the drift ratios of $2 \%$, $2.5 \%$ and $3.5 \%$. The deformed specimen after 3.5\% is shown in Figure 10(c). This is clear evidence supporting the scenario proposed in this study.

\section{Accuracy of FE analysis}

Figure 11(a) shows the comparisons of test results and FE analysis in terms of the relationship between the residual angle of rotation and drift ratio, and plots are made for Pairs 1, 5 and 9, which are regarded as the standard specimens for the respective levels. The simulated buckling initiation agreed well with the physical test, and the growth of torsional deformation was also simulated reasonably by the analysis up to an angle of rotation corresponding to the "off-one-thickness" criterion.

\section{STRENGTH, STIFFNESS AND ENERGY DISSIPATION}

\section{Yield strength}

To obtain the experimental yield strength $Q_{y t}$, two strain gauges glued on the surface of the link, Gauges 2 and 5 in Figure 8(ba), were monitored. The yield strength was determined as the shear force applied to the specimen when one of the strain gauges exceeded the yield strain obtained from the coupon test. The yield strengths obtained are listed in Table 2, together with the analytical strength estimated using Equation (3). In this table, Pairs 4 and 8 are not included as those specimens had rectangular links. Pair 10 is also excluded the specimens had links with different width-thickness ratios. The experimental and analytical strengths were very similar (with errors no greater than 5\%) for Pairs 5, 6, 7 and 9 (those for Levels 2 and 3), while the experimental strength was smaller by 15 to $25 \%$ than the analytical strength for Pairs 1, 2 and 3 (those for Level 1). The links in Level 1 were thinner 
than those in Levels 2 and 3; hence they were more susceptible to local buckling before significant yielding, which was thought to be the major cause of the discrepancy.

The local buckling stress of the double-tapered links was estimated. It was impractical to derive a closed-form equation for the exact elastic buckling load of double-tapered links because of the tapered shape, so the double-tapered link was assumed to be equivalent to a rectangular link with a uniform width of $b=2 a$, the width at the quarter-height section. The local buckling stress of a rectangular plate is given by [25]:

$$
\sigma_{c r}=k \frac{\pi^{2} E}{12\left(1-v^{2}\right)(b / t)^{2}},
$$

where $k$ is the buckling coefficient determined by the plate geometry and boundary conditions; $E$ and $v$ are Young's modulus and Poisson's ratio; $b$ is the plate width; and $t$ is the thickness. The buckling strength $Q_{c r}$ under this critical stress is estimated as follows:

$$
Q_{c r}=\frac{8 a^{2} t}{3 H} \sigma_{c r}
$$

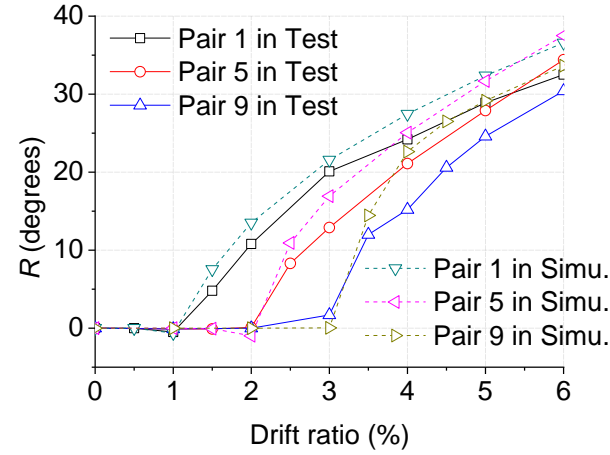

(a)

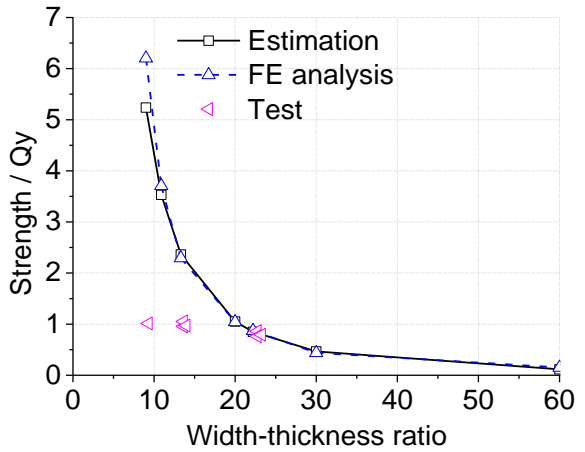

(b)

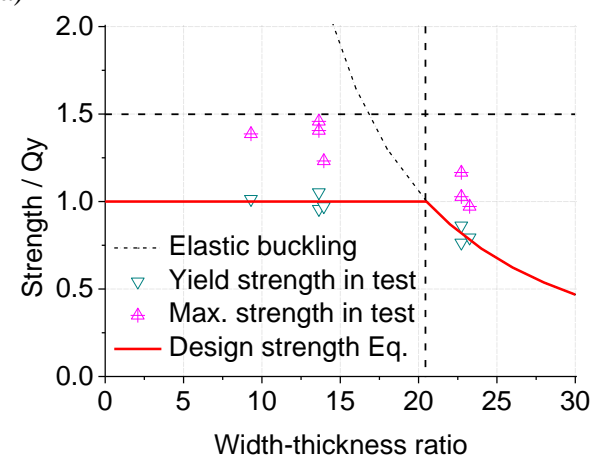

(c)

Figure 11 Discussion for test results: (a) comparisons of angle of rotation versus drift ratio 
between test and simulation; (b) buckling strength validation; (c) proposed design strength.

Considering the stress distribution induced in the link, the boundaries with three edges simply supported and one edge free were adopted. With this boundary condition, the buckling coefficient $k$ when subjected to pure bending is 0.85 [26]. The local buckling strength estimated with Equation (9) and the lateral torsional buckling strength estimated using Equation (6) are included in Table 2. For Pairs 1 to 3, the local buckling strength was smaller than the corresponding lateral torsional buckling strength. Thus, local buckling was the likely failure mode for these specimens. For Pairs 5 to 9 , the predicted values for two buckling strengths were significantly greater than unity, thus the specimens were subject to significant yielding prior to the onset of buckling. While it was difficult to estimate the exact buckling strength in the inelastic range, the failure mechanism in terms of buckling is explained as follows. The most critical section was the difference between local buckling and lateral torsional buckling, that is, the former at the quarter-height and the latter at the link end. Although the end section remained elastic, the quarter-height section was subjected to significant yielding with the taper of the links. In Table 2, while the critical strengths for lateral torsional buckling were similar or slightly smaller than those for local buckling in Pairs 5 to 9, the local buckling strength significantly decreased with the growth in yielding at the quarter-height section and thus the governing failure mode remained as local buckling. This reasoning was further confirmed because the experimental buckling shape was the same as for local buckling in all the specimens.

Figure 11(b) shows the relationship between the buckling force (normalized by $Q_{y}$ ) and width-thickness ratio, one obtained from Equation (9) with $k=0.85$ and the other from

Table 2 Summary of test results

\begin{tabular}{c|c|c|c|c|c|c|c}
\hline Pair & $Q_{y t}(\mathrm{kN})$ & $Q_{y}(\mathrm{kN})$ & $Q_{y t} / Q_{y}$ & $Q_{c r} / Q_{y}$ & $Q_{t t} / Q_{y}$ & $Q_{\max t}(\mathrm{kN})$ & $Q_{\max t} / Q_{y}$ \\
\hline 1 & 11.8 & 13.7 & 0.86 & 0.82 & 0.84 & 16.0 & 1.17 \\
\hline 2 & 15.7 & 20.6 & 0.76 & 0.82 & 1.41 & 21.1 & 1.03 \\
\hline 3 & 85.1 & 107.2 & 0.79 & 0.78 & 1.98 & 104.2 & 0.97 \\
\hline 5 & 15.7 & 16.5 & 0.96 & 2.26 & 1.28 & 23.1 & 1.41 \\
\hline 6 & 26.0 & 24.7 & 1.05 & 2.26 & 2.06 & 36.0 & 1.46 \\
\hline 7 & 62.5 & 64.3 & 0.97 & 2.16 & 2.81 & 79.2 & 1.23 \\
\hline 9 & 40.6 & 40.0 & 1.01 & 4.87 & 3.84 & 55.5 & 1.39 \\
\hline Pair & $K_{t}$ & $K$ & $K_{t} / K$ & $K_{\bmod }$ & $K_{t} / K_{\bmod }$ & & \\
\hline 1 & $14 N / \mathrm{mm})$ & $(\mathrm{kN} / \mathrm{mm})$ & & $(\mathrm{kN} / \mathrm{mm})$ & & \\
\hline 2 & 32.0 & 52.3 & 0.61 & 33.1 & 0.93 & & \\
\hline 3 & 110.5 & 206.7 & 0.53 & 176.6 & 0.62 & & \\
\hline 5 & 12.0 & 13.7 & 0.88 & 13.4 & 0.89 & & \\
\hline 6 & 34.8 & 43.8 & 0.79 & 39.3 & 0.88 & & \\
\hline 7 & 63.3 & 94.7 & 0.67 & 87.9 & 0.72 & & \\
\hline 9 & 33.1 & 43.0 & 0.77 & 41.3 & 0.80 & & \\
\hline & & & & & &
\end{tabular}


the FE analysis conducted for double-tapered links with various width-thickness ratios. The correlation between the two forces (for the same width-thickness ratio) was very reasonable, which justified using $k=0.85$. Table 2 also lists the estimated buckling strength $Q_{c r} / Q_{y}$, which was close to the experimental yield strength $Q_{y t} / Q_{y}$ for specimens in Level 1, with a difference within $6 \%$. This and a previous observation on the yield strength indicates that the design strength of the double-tapered link can be estimated reasonably by the smaller of the strengths calculated from Equations (3) and (9), as shown in Figure 11(c).

\section{Estimation of the maximum strength}

The maximum strength obtained from the test is also listed in Table 2 and plotted in Figure 11(c). Here, the maximum strength $Q_{\max t}$ was defined as the largest absolute strength obtained up to the completion of the $6 \%$ drift ratio cycles. The maximum ratio of $Q_{\max t} / Q_{y}$ was never greater than 1.5, which indicated that the plastic strength $Q_{p}$ in Equation (4) can be conservatively used in the estimation of maximum strength.

\section{Estimation of elastic stiffness}

The elastic stiffness of the individual links is given in Equation (5). Assuming that the end zones are rigid, the elastic stiffness of the specimens is the summation of the stiffness of the individual links. The estimated value $K$ is listed in Table 2, together with the elastic stiffness obtained experimentally. The correlation was not necessarily so reasonable. In particular, the discrepancy was more significant in Pairs 2 and 6, in which the end zones were larger (at a depth of 110 and $60 \mathrm{~mm}$ for the others). This implies the effect of flexibility of the end zones was not negligible. Therefore, the stiffness of the end zone $K_{\mathrm{c}}$ was estimated using Equation (10) to account for the flexural and shear deformations of the end zone. Note that the end zone in Equation (10) refers to the entire end zone of the panel, taken as a continuous rectangular plate. The modified stiffness $K_{\bmod }$ incorporating the stiffness of the end zone, was also estimated in Table 2 . The difference between the test and estimation was significantly smaller with a difference not greater than $12 \%$ for the specimens made of a $2.2 \mathrm{~mm}$ plate (Pairs 1, 2, 5, and 6). The specimens made of a $4.3 \mathrm{~mm}$ plate (Pairs 3, 7, and 9) still had relatively large differences from $20 \%$ to $38 \%$.

$$
K_{C}=\frac{1}{\int_{0}^{c}\left(\frac{h}{2}+x\right) d x+\int_{0}^{c} \frac{1.2}{G A(x)} d x}=\frac{1}{\frac{c\left(3 h^{2}+6 h c+4 c^{2}\right)}{\mathrm{E} t B^{3}}+\frac{1.2 c}{G B t}}
$$

where $c$ is depth of the end zone; $A$ is the sectional area of the end zone and $B$ is the total width of the entire end zone.

\section{Energy dissipation behavior}

The shear force versus drift ratio relationship is shown in Figure 12(a) for Pair 10 with the combination of links for Levels 1 to 3 . The strength started to decrease at $2.0 \%$ with the onset of local buckling in the link for Level 1. The decrease in strength was not very significant and the shear wall sustained the average strength (for positive and negative loading directions) larger than $80 \%$ of the yield strength $Q_{y}$. The equivalent damping ratios 


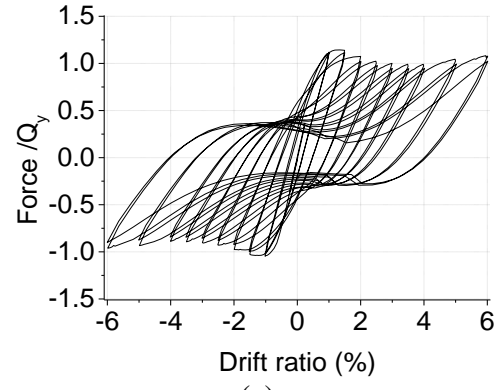

(a)

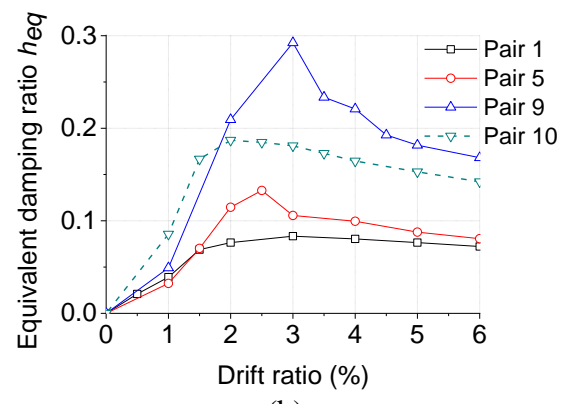

(b)

Figure 12 Hysteresis and equivalent damping ratio: (a) hysteresis of Pair 10; (b) equivalent damping ratios.

estimated using the standard procedure [27] for Pairs 1 (Level 1), 5 (Level 2) and ${ }_{2}-9$ (Level 3 ), and for Pair 10 are plotted for each drift ratio in Figure 12(b), in which one loop in the second cycle was used for the calculation. As elastic local buckling occurred in Level 1 (Pair 1), the hysteresis naturally involved significant pinching; hence the damping ratio remained small. In Level 2 (Pair 5), serious torsional deformation after local buckling did not occur until 2\%; hence the loops below the drift ratio were rather fat, with the damping ratio in a range of over 0.1. In Pair 9 (Level 3), stable hysteresis was obtained up to a drift ratio of $3 \%$. Pinching and corresponding reduction in the damping ratio occurred for cycles with drift ratios of $3.5 \%$ or greater. In Pair 10, pinching became significant after $2.5 \%$ in drift ratio and the damping ratio decreased gradually for larger drift ratios.

\section{CONCLUSIONS}

This paper presented a novel slit shear wall with double-tapered links that functioned as a hysteretic damper and as a condition assessment device. The number of torsionally deformed links, each of which was designed to rotate at specified drift ratios, was used as an indicator to estimate the maximum experienced deformation of the shear wall. Relying solely upon the inspection by naked eyes, we are able to judge immediately after the earthquake event whether or not the deformation at certain stories of the building where the proposed shear walls are deployed exceeds the deformation permitted in design. The major findings from numerical simulations and associated experiments are summarized as follows:

(1) To achieve notable torsional deformation for condition assessment, the link with a double-tapered shape was adopted. In the experiment, all specimens with double-tapered links exhibited large ductility, without any fracture observed below a drift ratio of $6 \%$. The growth of out-of-plane buckling was enhanced by $50 \%$ relative to the corresponding rectangular links at a drift ratio of $6 \%$.

(2) Buckling of double-tapered links was controlled primarily by the width-thickness ratio and was little affected by the aspect ratio. Using the results of numerical simulations, the width-thickness ratios of 22.7, 13.6 and 9.3 were selected for achieving notable torsional 
deformation at the drift ratios of 1.5\%, 2.5\% and 3.5\% (Levels 1, 2 and 3), respectively.

(3) The test resulted in success with most links notably buckled and reached the preset "offone-thickness" criterion at the target drift ratios of Levels 1 to 3 . The specimen featured with a combination of three different double-tapered links for Levels 1 to 3 showed clear torsional deformation at the drift ratios of $2 \%, 2.5 \%$ and $3.5 \%$-drift ratios. This was a clear evidence for supporting the scenario of condition assessment proposed in this study.

(4) The design strength matched very well with the yield strength obtained in the test; the maximum strength was close to the yield strength for the thinner specimens (Level 1) and about 1.4 times the yield strength for the thicker specimens (Levels 2 and 3). The experimental elastic stiffness was $82 \%$ on average with respect to the design stiffness. The influence of end-zone flexibility was found non-negligible for stiffness estimation.

(5) In future work, a general design guideline needs to be established. A design equation with the width-thickness ratio as the primary design variable is desirable to predict the drift ratio of the link corresponding to notable torsional deformation. The loading protocol adopted here was the one commonly used to evaluate the seismic performance of shear walls. Tests with other loading protocols should be conducted to investigate the sensitivity of the proposed scenario of condition assessment with respect to the non-stationary characteristics of earthquake-type loading.

\section{ACKNOLEDGEMENTS}

The present work is partially supported by the 5th Seismic Isolation and Response-Control research grant award of Japan Society of Seismic Isolation (PI: Masahiro Kurata). Our sincere thanks go to Dr. Kazuhiro Hayashi, Mr. Kei Fukihara, and Mr. Yudai Taniguchi for their assistance offered in the experimental work.

\section{REFERENCES}

[1] Loh, C-H., Chao, S-H., Weng, J-H., and Wu T-H. Application of subspace identification technique to long-term seismic response monitoring of structures, Earth. Eng. Struct. Dyn. 2014; [posted online ahead of printing]. DOI: 10.1002/eqe.2332.

[2] Limongelli, M. P. Seismic health monitoring of an instrumented multistory building using the interpolation method, Earth. Eng. Struct. Dyn. 2014; 43(11): 1581-1602.

[3] Kurata, M., Li, X., Fujita, K., Yamaguchi, M. Piezoelectric dynamic strain monitoring for detecting local seismic damage in steel buildings, Smart Mater. Struct. 2013: 22, 115002. DOI:10.1088/0964-1726/22/11/115002.

[4] Benavant-Climent, A., Morillas, L., Escolano-Margarit, D. Seismic performance and damage evaluation of a reinforced concrete frame with hysteretic dampers through shake-table tests, Earth. Eng. Struct. Dyn. 2014; [posted online ahead of printing]. DOI: 10.1002/eqe.2459.

[5] Kumar, M., Whittaker, A.S., Constantinou, C. An advanced numerical model of elastomeric seismic isolation bearings, Earth. Eng. Struct. Dyn. 2014; 43(13): 19551974. 
[6] Shi, Y., Kurata, M., Nakashima, M. Disorder and damage of base-isolated medical facilities when subjected to near-fault and long-period ground motions, Earth. Eng. Struct. Dyn. 2014; 43(11): 1683-1701.

[7] Takeuchi, T., Ozaki, H., Matsui, R., and Sutcu, F. Out-of-plane stability of bucklingrestrained braces including moment transfer capacity, Earth. Eng. Struct. Dyn. 2014; 43(11): 851-869.

[8] American Institute of Steel Construction: Seismic provisions for structural steel buildings. Chicago, Illinois [2010].

[9] Canadian Standard Association: CSA S16-09: Design of Steel Structures (7th edition). Toronto, Canada [2009].

[10] Roberts, T. M. and Ghomi, S. Sabouri. Hysteretic Characteristics of Unstiffened Plate Shear Panels. Thin-Walled Struct. 1991; 12(2): 145-162.

[11] Vian, D. Steel plate shear walls for seismic design and retrofit of building structures. Ph.D. dissertation. SUNY at Buffalo: Buffalo, New York, 2005.

[12] Qu B, Bruneau M, Lin CH, Tsai KC. Testing of full-scale two-story steel plate shear wall with reduced beam section connections and composite floors. J. Struct. Eng. 2008; 134(3): 364-73.

[13] Kurata, M., Leon. T. R., DesRoches, R., and Nakashima, M. Steel Plate Shear Wall with Tension-Bracing for Seismic Rehabilitation of Steel Frames. J. Const. Steel Research 2012; 71: 92-103.

[14] Martinez-Rueda, J. E. On the evolution of energy dissipation devices for seismic design. Earthquake Spectra 2002; 18(2): 309-346.

[15] Hitaka, T., Matsui, C., Tsuda, K., and Yanagida, Y. Elasticplastic behavior of building steel frame with steel bearing wall with slits. Proc. 12th WCEE 2000; 833/6/A.

[16] Hitaka, T. and Matsui, C. Experimental study on steel shear wall with slits. J. Struct. Eng. 2003; 129(5): 586-595.

[17] Cortes G. and Liu J. Experimental evaluation of steel slit panel frames for seismic resistance. J. const. steel research 2011; 67: 181-191.

[18] Jacobsen A., Hitaka T. and Nakashima M. Online test of building frame with slit wall dampers capable of condition assessment. J. const. steel research 2010; 66: 13201329.

[19] Jacobsen, A. Development of steel slit wall dampers with embedded condition assessment capabilities. Ph.D. dissertation. Kyoto Univ.: Kyoto, 2010.

[20] Okamura, T., Kurata, M., and Nakashima, M. Development of Slitted Steel Shear Walls Capable of Detecting Damage States. Proc. 15th WCEE 2012.

[21] Ma X., Borchers E., Peña A., Krawinkler H., Billington S. and Deierlein G. Design and behavior of steel shear plates with openings as energy-dissipating fuses. Report No. 173, Stanford University, 2010.

[22] Dassault Systèmes., ABAQUS Ver. 6.10 User's Manual. http://www.abaqus.com

[23] Trahair N.S. Flexural-Torsional Buckling of Structures. CRC press: Boca Raton, 1993.

[24] Bradford, M. and Cuk, P. Elastic Buckling of Tapered Monosymmetric I - Beams. J. 
$611 \quad$ Struct. Eng. 1988; 114(5): 977-996.

[25] Timoshenko S.P. and Gere J.M. Theory of elastic stability: 2nd Edition. McGrawHill: New York, 1961.

[26] Johnston B.G. Guide to stability design criteria for metal structures (6th edition). John Wiley \& Sons, Inc: New York, 2010.

[27] Chopra., A. K. Dynamics of Structures: Theory and Applications to Earthquake Engineering (2nd Edition). Prentice Hall: New Jersey, USA, 2000. 